\title{
Minimização de Ruído Eletroquímico Usando \\ Processamento Digital de Sinais
}

\section{Electrochemical noise minimization using digital signal processing}

\author{
Paulo Rogério Scalassara1; Claudia Smaniotto Barin²; Carlos Dias Maciel ${ }^{3}$
}

Resumo

\begin{abstract}
Neste trabalho, apresenta-se uma discussão sobre ruídos eletroquímicos presentes em sinais de baixa amplitude e revisão bibliográfica de métodos de filtragem de sinais para diminuir a interferência desses ruídos. Um desses métodos, chamado Análise de Espectro Singular (SSA), é descrito com mais detalhes e utilizado para filtrar alguns sinais obtidos em experimentos de deposição eletrostática. Mostram-se, também, comparações dos resultados de outras técnicas de filtragem, como média móvel, SavitskyGolay e Transformadas de Fourier e Wavelet, apresentando-se as suas vantagens e desvantagens. Este estudo mostra que o método SSA é eficiente e de fácil aplicação nos sinais obtidos, sendo os resultados de extrema importância para a compreensão das características da eletrodeposição. Na seção Introdução, mostra-se a origem dos sinais e discute-se a deposição elétrica usando microeletrodos, suas vantagens, problemas e técnicas para filtragem dos ruídos. Na seção Teoria, o método SSA é descrito, mostrando suas vantagens e razões para o uso com sinais ruidosos. Em Materiais e Métodos, tem-se uma sucinta descrição dos equipamentos e software usados para obtenção e processamento dos sinais. Em Resultados, apresentam-se comparações dos sinais reconstruídos por SSA com aqueles obtidos por outras técnicas de filtragem, sendo os comentários finais apresentados na seção Conclusão.
\end{abstract}

Palavras-chave: Processamento digital de sinais. Microeletrodo. Ruído eletroquímico.

\begin{abstract}
This work discusses electrochemical noise in low amplitude signals and reviews the bibliography on signal filtering methods used to reduce noise interference. One of these methods, the Singular Spectrum Analysis (SSA), is described in detail and used to filter some signals in electrostatic deposition experiments. This study also compares results from other filtering techniques such as moving average, Stavitsky-Golay and Fourier and Wavelet, and discusses the advantages and disadvantages of transforms. Results have shown that the SSA method is efficient, of easy applicability, and extremely important for the understanding of electrodeposition characteristics. In the Introduction of this work, the origin of the signals is discussed, and the advantages, problems and the noise filtering techniques related to electrical deposition through microelectrodes are presented. In the Theory section, the SSA method is described, and the reasons for using it with noisy signals are presented. In the Materials and Methods section, the equipment and software used for collecting and processing the signals are described briefly. Finally, in the Results section, the signals reconstructed through SSA, as well as those found in other filtering techniques are presented.
\end{abstract}

Key words: Signal processing. Microelectrode. Electrochemical noise.

1 Aluno de Mestrado e bolsista CNPq do Dept ${ }^{\circ}$ Engenharia Elétrica da Universidade Estadual de Londrina

2 Professora Convidada do Dept ${ }^{\circ}$ Engenharia Elétrica da Universidade Estadual de Londrina. E-mail: cbarin@uel.br

3 Docente Visitante do Dept ${ }^{\circ}$ Engenharia Elétrica da Universidade Estadual de Londrina 


\section{Introdução}

A deposição de metais e ligas tem apresentado elevado interesse tanto na área científica quanto tecnológica, e a natureza do produto formado é dependente do potencial aplicado, o $\mathrm{pH}$ do meio e outros parâmetros tais como: temperatura, agitação da solução, concentração das espécies depositáveis e concentração de agentes complexantes. Embora estes parâmetros sejam de difícil controle e/ou escolha, eles têm uma marcante influência nas características finais dos depósitos.

Esses depósitos podem ser obtidos por uma infinidade de técnicas, como, por exemplo: deposição química, deposição autocatalítica (eletroless), fusão metálica, deposição química a vapor, entre outras. Entretanto, do ponto de vista econômico, a eletrodeposição é uma das formas mais interessantes para a realização desses processos.

Um aspecto fundamental do processo de eletrodeposição é o estágio inicial da formação de fase, normalmente chamada eletrocristalização (BOCKRIS; RAZUMNEY, 1967). Esse estágio é normalmente associado a um processo de crescimento de duas ou três dimensões onde o número de núcleos formados na superfície do eletrodo é fortemente dependente do sobrepotencial aplicado (SOUTHAMPTON ELECTROCHEMISTRY GROUP, 1995, BENTO; MASCARO, 2002). Microeletrodos são alternativas atrativas para o estudo dos passos iniciais da deposição.

Eletrodos com dimensões de micrometros eram pouco utilizados até a década de 80 , mas o interesse nestes eletrodos cresceu exponencialmente na última década. Há dez anos atrás, poucos reconheciam as vantagens do uso de eletrodos de pequenas dimensões, mas hoje a literatura é vasta em publicações que auxiliam a compreensão das propriedades dos microeletrodos (MEs) e também que demonstram novos tipos de aplicações.

Algumas áreas da eletroanalítica e da cinética eletródica tornaram-se objeto de numerosos trabalhos, como a eletroanálise in vivo (WIGHTMAN; EWING; DAYTON, 1981) ou em soluções sem eletrólito de suporte (SILVA et al., 1994), eletrodeposições de núcleos isolados (SCHARIFKER; HILLS, 1981), voltametria em alta velocidade de varredura (ROBINSON; MCCREERY, 1981) e em eletrólitos pouco condutores (WIPF; WIGHTMAN, 1990), eletrocatálise de reações envolvendo desprendimento de gases (TAVARES et al., 1992), entre outros.

Devido às suas pequenas dimensões, geralmente entre 0,1 e $50 \mathrm{~mm}$ os MEs apresentam particularidades como:

a) Alta eficiência no transporte de massa devido à forma esférica da camada de difusão criada ao seu redor, sendo comparáveis a macro-eletrodos. Isso facilita o estudo de reações eletródicas rápidas sob condições de estado estacionário e nãoestacionário, que é atingido em tempos muito mais curtos do que em eletrodos convencionais, e a detecção de intermediários em reações orgânicas que possuam tempo de vida muito curto.

b) A capacitância da dupla camada é reduzida devido ao decréscimo da área superficial, possibilitando o emprego de MEs em velocidades de varredura maiores que $10 \mathrm{kV} \cdot \mathrm{s}^{-1}$, sem a ocorrência de distorções nas respostas obtidas.

c) As correntes medidas nos MEs são extremamente pequenas (ordem de nano a picoampères) e não alteram significativamente a concentração da espécie eletrolisada na solução. Devido a esta característica, as respostas dos MEs são muito pouco sensíveis aos erros devidos a queda ôhmica, podendo ser usados em soluções de alta resistência e na ausência de eletrólito de suporte.

Entretanto, essa última vantagem, às vezes, apresenta um pequeno inconveniente: $\mathrm{o}$ aumento do ruído no sinal, pois a diminuição do tamanho do eletrodo faz com que a corrente medida seja da ordem de nanoampères ou, até mesmo, picoampères. Isso ocorre, principalmente, se o amplificador usado para dar ganho no sinal não for adequado para as amplitudes de correntes e tensões usadas. Uma alternativa para esse problema é o uso de técnicas de processamento digital de sinais. 
Outras vantagens do emprego de MEs são: miniaturização de células, uso de sistemas com apenas dois eletrodos (o eletrodo de referência atua como auxiliar), decréscimo do custo do material e simplificação da instrumentação.

No contexto de processamento digital de sinais, a eliminação de ruído buscando uma melhor análise dos dados é chamada filtragem. Os ruídos principais que afetam os dados de baixa amplitude coletados por instrumentos de gravação são (CERQUEIRA et al., 2000): Johnson ou ruído térmico, Shot e Flicker. Métodos comumente usados para remoção desses ruídos são: média móvel e Savitsky-Golay, que são de fácil implementação, mas podem distorcer os dados, comprometendo a análise (CERQUEIRA et al., 2000; CHOW et al., 1995). As principais distorções nos sinais são devidas à diminuição de picos e alargamento de suas bases, as quais se intensificam com o aumento do tamanho da janela usada no filtro (CHOW et al., 1995).

Em trabalhos recentes publicados no campo de eletroquímica, métodos que atuam diretamente sobre as frequiências que compõem o sinal têm obtido bons resultados, eles possibilitam a remoção seletiva de ruído. Entre esses, estão os filtros baseados em Transformadas de Fourier (TF) e Wavelet (TW), segundo Galvão et al. (2001), Pletcher (1991). Apesar da boa performance desses métodos, sabe-se, que algumas vezes, as transformações por eles empregadas podem ocasionar a compressão dos dados.

Como a filtragem de sinais utilizando essas transformações baseia-se na atenuação de parte do espectro de freqüências do sinal, quando o ruído possuir componentes em baixas freqüências, estes não serão removidos. Mas o maior problema surge quando o sinal possui componentes acima da freqüência de corte do filtro, pois essas são eliminadas, gerando distorção. Além disso, esses métodos requerem a escolha correta de parâmetros como: freqüência de corte para Transformada de Fourier e tipo de função mãe e número de níveis da decomposição para Transformada Wavelet (GALVÃO et al., 2001); sendo que, essa escolha geralmente não é trivial.
O objetivo deste artigo é mostrar um bom caminho para eliminação de ruído em sinais obtidos em microeletrodos e de métodos de cronoamperometria, utilizando uma técnica de processamento digital de sinais chamada Análise de Espectro Singular, melhorando, dessa forma, a qualidade dos dados experimentais.

\section{Teoria}

A técnica de processamento de sinais utilizada para remoção do ruído eletroquímico é a Análise de Espectro Singular (SSA - singular spectrum analysis). O SSA é um método usado para extrair informação de séries temporais de curta duração e com ruído, com a vantagem de não precisar de nenhum conhecimento prévio do sistema.

Este método baseia-se na aplicação univariável no domínio do tempo da análise de componentes principais (PCA - principal component analysis) (GHIL; KEPPENNE, 1992). É essencialmente um método de análise linear que pode ser aplicado a dinâmicas não-lineares, devido à característica dos autoelementos que se adaptam aos dados analisados.

Outros métodos de análise de espectro, como a Transformada de Fourier, usam funções básicas predefinidas para decompor os espectros de sistemas sendo, no caso da TF, senos e co-senos. Em SSA, são os próprios dados que determinam as funções de bases ortogonais que são ótimas estatisticamente.

A análise é executada no espaço vetorial de coordenadas de atraso de uma série temporal (VAUTARD; YIOU; GHIL, 1992). Primeiramente, considerando um sinal de tempo discreto $x$, criado, em teoria, pela amostragem de um sinal de tempo contínuo com período de amostragem $T_{s}$, Equação 1:

$$
x=\left(s_{1}, s_{2}, \ldots, s_{N}\right)^{T}
$$

sendo $s_{i}$ a $i$-ésima amostra, $N$ o número de pontos de dados e (.) ${ }^{\mathrm{T}}$ a operação de transposição matricial. 
Usando o método dos atrasos (BROOMHEAD; KING, 1986), é possível criar uma série temporal $x_{i}$ de comprimento $M, \operatorname{com} 1 \leq i \leq N$, dada pela Equação 2:

$$
x_{i}=\left(s_{i}, s_{i+1}, \ldots, s_{i+M}\right)^{T}
$$

A largura da janela dessa série é $T_{w}=M \cdot T_{s}$. Criase, então, a matriz de trajetórias, representada por $X$, sendo $N_{T}=N-(M-1)$, dada pela Equação 3:

$$
X=N_{T}^{-1 / 2}\left(x_{1}^{T}, x_{2}^{T}, \ldots, x_{N_{T}}^{T}\right)^{T}
$$

A expansão da série temporal $x_{i}$ em seus componentes principais (CPs) é dada pela Equação 4, sendo $a_{i}^{k}$ o k-ésimo CP e $E^{k}$ as funções ortogonais empíricas (EOF - empirical orthogonal functions) ou autovetores da matriz de covariância cruzada de $X$ :

$$
x_{i+j}=\sum_{k=1}^{M} a_{i}^{k} E_{j}^{k} \quad 1 \leq j \leq M
$$

Os autovalores $l_{i}$ da matriz de covariância são os valores de variância do $k$-ésimo $\mathrm{CP}$ e são normalmente organizados em ordem descendente (VAUTARD; YIOU; GHIL, 1992).

Essa característica é muito importante, pois permite que a expansão da série seja truncada, retendo somente a informação dos primeiros componentes principais. Em SSA, esses primeiros CPs contêm uma grande quantidade da variância do sinal, sendo os últimos basicamente variância de ruído (se este for ruído branco), conseqüentemente, é possível eliminar boa parte desse ruído do sinal.

Os componentes principais podem ser mais bem definidos considerando-os como coeficientes de projeção ortogonal da série original no correspondente EOF, como mostra a Equação 5:

$$
a_{i}^{k}=\sum_{j=1}^{M} x_{i+j} E_{j}^{k} \quad, 0 \leq i \leq N-M
$$

Considerando-se um subconjunto $S$ de autoelementos $k$ para reconstruir o sinal, define-se o $k$-ésimo componente de reconstrução (CR) como a reconstrução da série temporal quando o subconjunto $S$ consiste de um único índice $k$, chamado $x^{k}$. Considerando que os CRs satisfazem a propriedade aditiva, a série reconstruída é dada pela Equação 6:

$$
y=\sum_{k \in S} x^{k}
$$

$\mathrm{E}$, se o subconjunto consistir de todos os $M \mathrm{CRs}$, a série reconstruída é igual à série original, tendose, assim, a Equação 7:

$$
x=\sum_{k=1}^{M} x^{k}
$$

O princípio de remoção do ruído de sinais por SSA consiste no uso de um conjunto de CRs para gerar uma reconstrução. Usa-se, para essa reconstrução, um subconjunto $S$, que seja composto somente pelos primeiros CRs, os quais (como os CPs) possuem a maior parte da variância do sinal, e, assim, elimina-se uma boa quantidade de variância do ruído.

\section{Materiais e Métodos}

As medidas eletroquímicas foram realizadas usando uma célula Pyrex® de dois eletrodos, sendo um deles feito de $\mathrm{Ag} / \mathrm{AgCl}$ como referência/auxiliar combinado e um eletrodo de trabalho composto por um fio em forma de disco de Pt de $50 \mathrm{~mm}$ selado em vidro. Em todos os experimentos eletroquímicos foi usado um potenciostato-galvanostato EG\&G-PAR modelo 273A ligado a um microcomputador modelo 486, $66 \mathrm{MHz}$, com 16 megabytes de memória RAM, controlado pelo software M270. A Figura 1 mostra um diagrama de blocos da montagem experimental. 


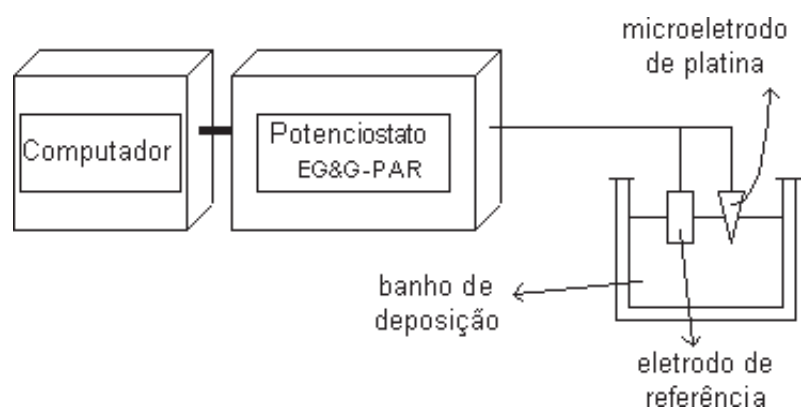

Figura 1. Diagrama de blocos ilustrando a montagem experimental utilizada nas medidas eletroquímicas

Visando à diminuição dos ruídos gerados por interferência do meio, presentes nos sinais obtidos, utilizou-se o princípio da gaiola de Faraday, pelo uso de uma caixa metálica ao redor do banho de deposição. Entretanto, os resultados obtidos não foram satisfatórios, ou seja, os ruídos não apresentaram diminuições significativas, portanto, o método foi descartado.

Para a aplicação de filtragem nos sinais pelo método da Análise de Espectro Singular, usou-se o Toolkit SSA-MTM (Singular Spectrum Analysis Multitaper Method) versão 4.2 para Linux, desenvolvido pelo grupo de Dinâmica Climática Teórica (Theoretical Climate Dynamics) do Departamento de Ciências Atmosféricas (Department of Atmospheric Sciences) e pelo Instituto de Geofísica e Física Planetária (Institute of Geophysics and Planetary Physics) da Universidade da Califórnia, Los Angeles (UCLA), (GHIL et al., 2002).

Esse software foi utilizado em um computador Pentium II, $333 \mathrm{MHz}$, com 128 megabytes de memória RAM, rodando RedHat Linux 8.0. Para a execução da análise pelo programa, fornecia-se a freqüência de amostragem dos sinais coletados nos experimentos e os arquivos com os dados, e para o resto dos parâmetros foram usados os valores padrões do programa. Assim, o software realizava a decomposição do sinal em seus componentes principais. As Figuras 2 e 3 mostram um exemplo do processo, usando um sinal obtido nos experimentos, com frequiência de amostragem igual a $1000 \mathrm{~Hz}$, sendo a primeira figura o sinal com ruído, e a segunda seus autovalores.

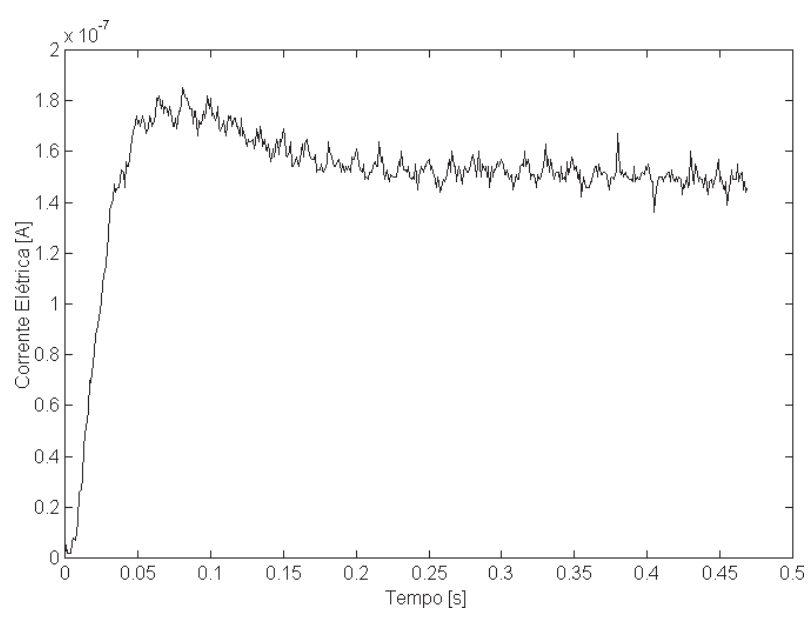

Figura 2. Exemplo do sinal eletroquímico com ruído, mostrando as grandes variações que atrapalham a análise dos resultados

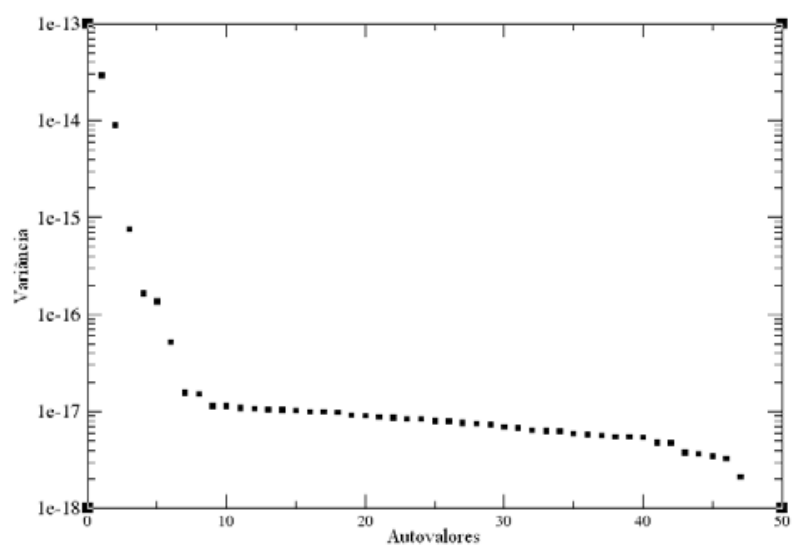

Figura 3. Decomposição do sinal ruidoso apresentado na figura anterior em seus componentes principais mostrando a diferença entre os dois primeiros componentes principais e o resto deles. Percebe-se que do segundo para o terceiro CP já existe uma distância de uma ordem de grandeza

Para a reconstrução, usavam-se somente os dois primeiros CPs, os quais têm uma contribuição muito maior para a variância do sinal do que os outros componentes, pois, para os casos analisados, entre o segundo e o terceiro $\mathrm{CP}$ existia uma diferença de mais de uma ordem de grandeza. Dessa forma, o sinal filtrado (reconstruído) possuía somente uma pequena 
parte da variância do ruído. O sinal reconstruído do exemplo anterior é apresentado na Figura 4, sendo a linha tracejada o sinal original e a linha cheia o sinal reconstruído.

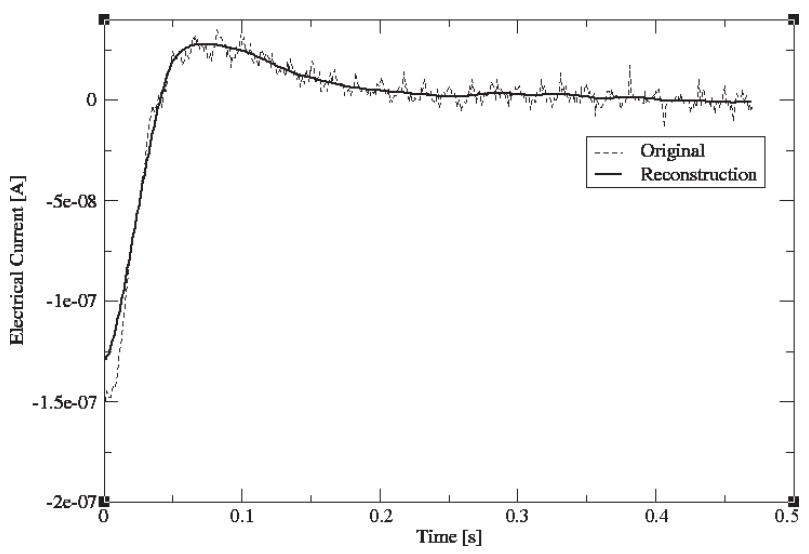

Figura 4. Comparação do sinal original (linha tracejada) com o sinal reconstruído (linha cheia) usando o dois primeiros componentes principais, mostrando a eliminação de boa parte do ruído

Comparando o sinal original e o reconstruído, observa-se que o último tem bem menos ruído. No sinal reconstruído, o valor médio do sinal foi removido pelo software. Além disso, existem algumas diferenças entre os valores iniciais dos sinais, principalmente porque o programa retira as oscilações de alta freqüência.

Para avaliar os resultados da filtragem por SSA, foram feitas algumas comparações simples com outros métodos. Foram realizadas algumas simulações usando-se Matlab 5.3 para Microsoft Windows em alguns com dos sinais analisados neste estudo. Os métodos de filtragem em questão foram por: média móvel, Savitsky-Golay, Transformada de Fourier e Transformada Wavelet.

Para a filtragem por média móvel, utilizou-se a Equação (8) para o algoritmo (OPPENHEIM; SCHAFER; BUCK, 1999), sendo M+N+1 o tamanho da janela usada na média:

$$
y[n]=\frac{1}{M+N+1} \sum_{k=-M}^{N} x[n-k]
$$

Nas simulações, usou-se tamanho da janela igual a 20, com 8 amostras para frente w 9 para trás. A filtragem Savitsky-Golay foi realizada utilizando-se uma função do Matlab, a qual suavizava um sinal de entrada utilizando um filtro polinomial gerado pelo método em questão. Para as simulações, usaram-se a ordem do polinômio igual a 3 e o tamanho da janela igual a 21 .

Para a filtragem usando Transformada de Fourier, projetou-se um filtro passa-baixa, com freqüência angular de corte igual a $0,7 \mathrm{p} \mathrm{rad} / \mathrm{s}$. Filtraram-se os espectros de potências dos sinais, por meio da convolução da resposta impulsiva do filtro com o sinal. A filtragem por Transformada Wavelet foi realizada utilizando-se funções pertencentes ao Matlab. Usaram-se dois níveis de decomposição e função mãe usada Daubechies 4.

\section{Resultados}

Os resultados das simulações com os outros métodos são apresentados nas Figuras 5 a 8 . A análise desses resultados é feita pela observação da quantidade de ruído eliminado do sinal. Pode-se ver que para a filtragem por média móvel, Figura 5, a quantidade de ruído eliminada é considerável, mas, segundo a literatura pesquisada (CERQUEIRA et al., 2000; CHOW et al., 1995), esse método também gera distorção nos dados. Para a filtragem Savitsky-Golay, os dados não sofrem tanta distorção, mas em compensação, a quantidade de ruído eliminada é bem inferior, Figura 6 .

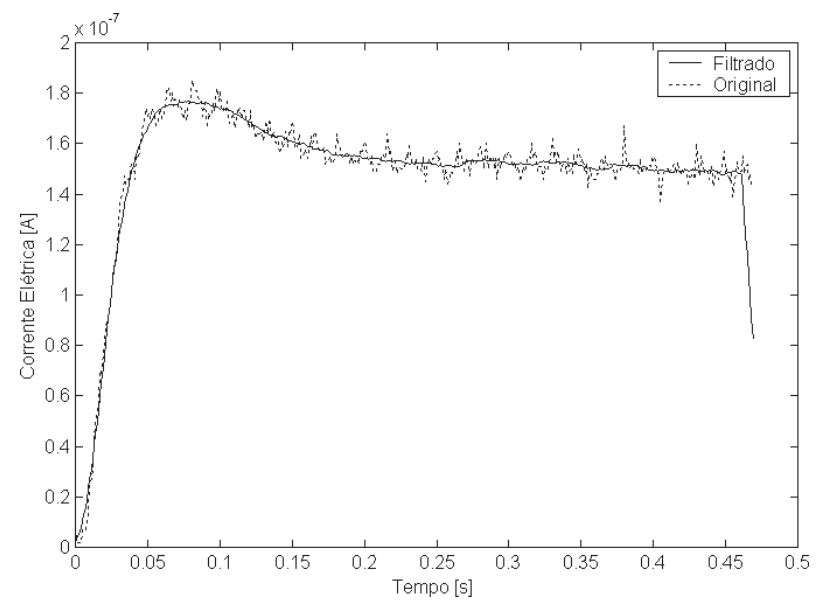

Figura 5. Sinal filtrado usando o método de média móvel, com janela de 20 pontos, sendo o sinal original feito com linha tracejada e o sinal filtrado com linha cheia 


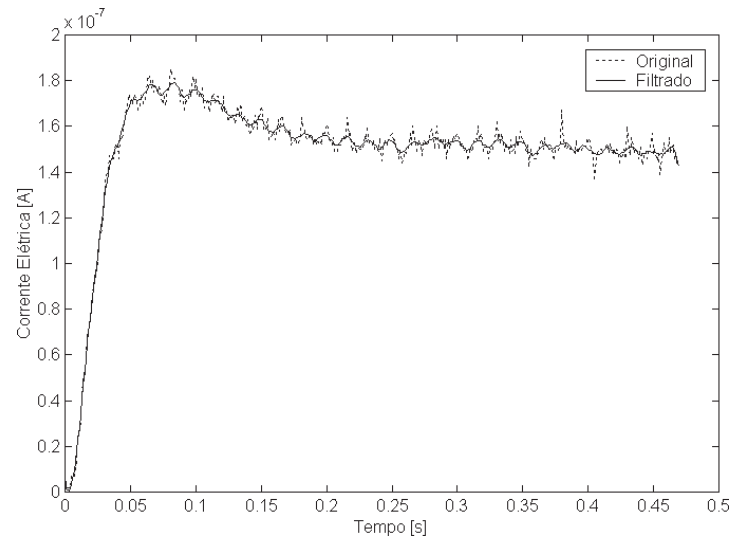

Figura 6. Sinal filtrado usando o método de Savitsky-Golay, com ordem do polinômio igual a 3 e tamanho da janela igual a 21 pontos, sendo o sinal original feito com linha tracejada e o sinal filtrado com linha cheia

Para a filtragem por TF, Figura 7, o sinal ficou com muito pouco ruído, mas ocorreu uma deformação visível dos dados, o que se deve, provavelmente, a informações do sinal que possuíam frequiências altas que foram retiradas pela filtragem. A filtragem por Transformada Wavelet apresentou resultados com grande quantidade de ruído ainda presente no sinal, Figura 8. Os resultados para os métodos que usam o espectro de frequiência na filtragem (TFe TW) são especialmente difíceis de serem avaliados, pois dependem de uma escolha adequada dos seus parâmetros. Os ensaios realizados neste trabalho não têm por objetivo descartar esses métodos, mas sim mostrar que o SSA possui algumas facilidades com relação a eles.

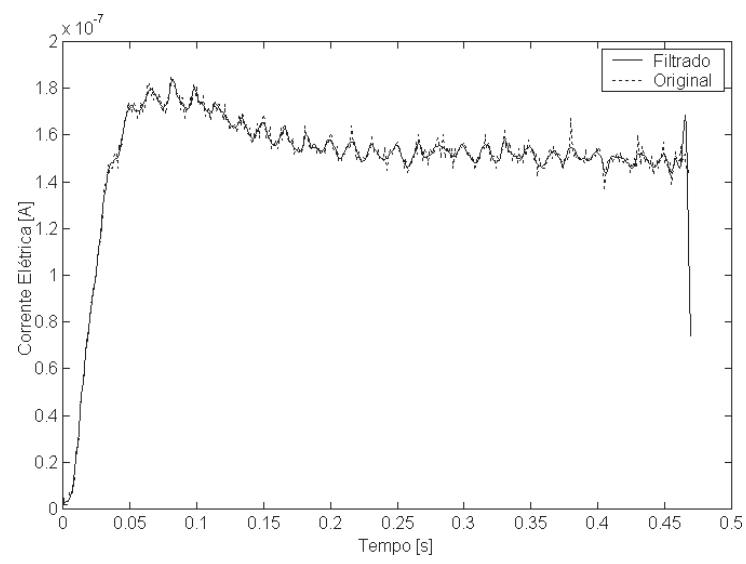

Figura 7. Sinal filtrado usando o método de Transformada de Fourier, usando um filtro passa-baixa no espectro de potências do sinal com freqüência angular de corte igual a $0,7 \mathrm{p} \mathrm{rad} / \mathrm{s}$, sendo o sinal original feito com linha tracejada e o sinal filtrado com linha cheia

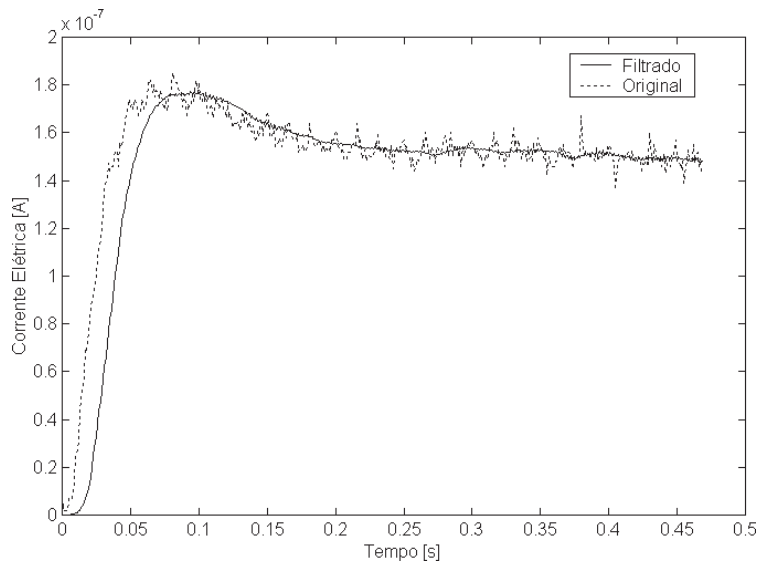

Figura 8. Sinal filtrado usando o método de Transformada Wavelet, com dois níveis de decomposição e função mãe Daubechies 4, sendo o sinal original feito com linha tracejada e o sinal filtrado com linha cheia

Com o uso do método SSA, os resultados foram muito significativos, pois grande parte do ruído eletroquímico foi retirada, como se pôde ver na Figura 4. Assim como as outras técnicas de processamento de sinais citadas, o SSA também apresenta desvantagens, pois a derivação teórica desse método considera que o ruído presente no sinal é branco, ou seja, possui contribuições iguais em todo o espectro de potências (ALLEN; SMITH, 1996). E, também, considera que o sinal seja estacionário em sentido amplo, portanto, quando se utiliza o método para separar o ruído do sinal desejado, gerase um pouco de distorção também. Mas, geralmente, o maior problema é a dificuldade de se escolher os componentes necessários para a reconstrução, o que não é o caso deste estudo, pois o ruído presente nos sinais se aproximava de ruído branco.

Pelo uso do SSA, vários sinais foram filtrados, sendo, em seguida, utilizados para análise dos resultados dos experimentos de deposição eletrostática. Buscando-se avaliar a forma de crescimento dos núcleos sobre a superfície eletródica, classificando-o em progressivo ou instantâneo. Esses dados são extremamente importantes para compreensão das características finais dos eletrodepósitos.

Como forma ilustrativa dos resultados obtidos com uso do SSA nestes sinais de cronoamperometria, 
mais dois exemplos da filtragem são apresentados nas figuras a seguir. As Figuras 9 e 10 mostram sinais com pouco e muito ruído, respectivamente. Como se pode ver, para ambos os casos, os resultados foram satisfatórios, ou seja, foi possível retirar grande quantidade do ruído eletroquímico presente nos sinais. Nas figuras, o sinal original foi feito em linha tracejada e o reconstruído, a partir dos dois primeiros componentes principais, em linha cheia. A frequiência de amostragem dos sinais é 500 e $225 \mathrm{~Hz}$ respectivamente, sendo estes parâmetros fornecidos ao software de análise.

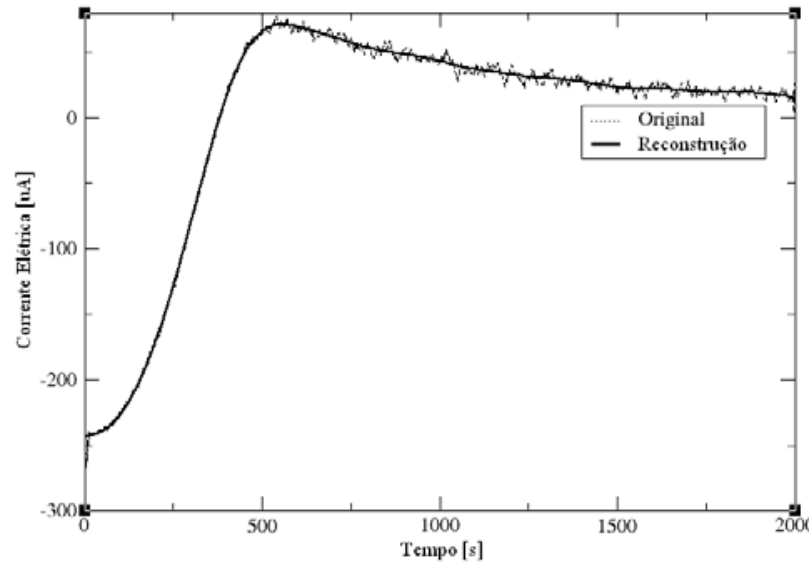

Figura 9. Exemplo de sinal com pouco ruído eletroquímico (linha tracejada). O sinal filtrado (linha cheia) foi obtido usando SSA, sendo reconstruído a partir dos dois primeiros componentes principais. A frequiência de amostragem é $500 \mathrm{~Hz}$.

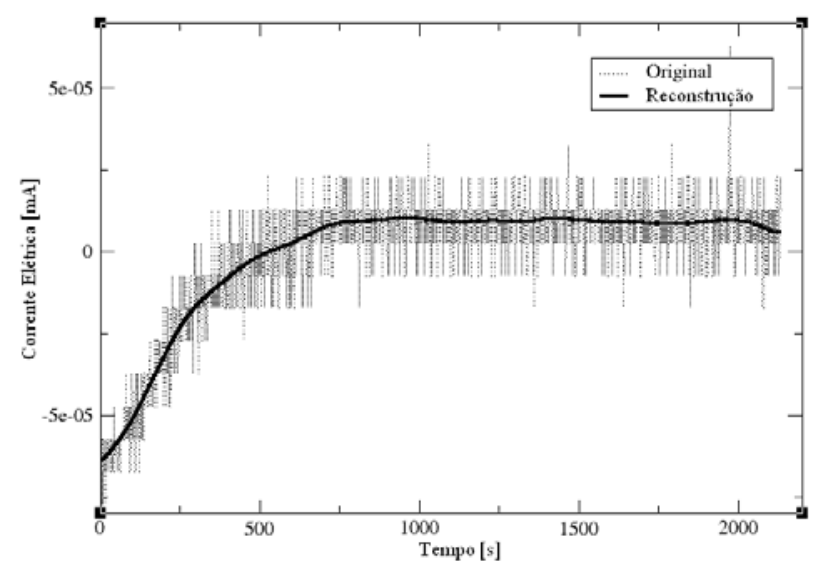

Figura 10. Exemplo de sinal com muito ruído eletroquímico (linha tracejada). O sinal filtrado (linha cheia) foi obtido usando SSA, sendo reconstruído a partir dos dois primeiros componentes principais, pode-se ver que grande parte do ruído foi eliminado. A freqüência de amostragem é $225 \mathrm{~Hz}$

\section{Conclusão}

Neste trabalho foram apresentadas várias vantagens do uso de microeletrodos no estudo de deposição eletrostática, e também um problema resultante, a baixa amplitude das correntes e tensões utilizadas, as quais implicam em maior presença de ruído de vários tipos no sinal. Com isso, a análise desses sinais fica muito afetada. Foi realizada uma revisão bibliográfica buscando soluções para esse problema, e foram descartados os métodos de filtragem com implementações mais simples, como média móvel e Savitsky-Golay, os quais podem distorcer os dados ao eliminar uma parcela do ruído.

Outras técnicas de processamento digital de sinais, as quais em outros trabalhos obtiveram bons resultados, foram também consideradas, como filtragem por Transformada de Fourier e Transformada Wavelet. Verificou-se, porém, que essas transformações poderiam gerar distorção dos dados por filtrarem componentes de frequiência dos sinais desejados. Além disso, seus parâmetros de filtragem são de difícil escolha, o que aumenta a complexidade desses métodos.

Continuando na linha do processamento digital de sinais, foi feito um estudo acerca do método chamado Análise de Espectro Singular, que apresenta bons resultados segundo os artigos pesquisados e poucos efeitos adversos aos sinais. O SSA possui várias vantagens: não precisa de conhecimento prévio do sistema em estudo, é basicamente um método de análise linear e possui uma característica de adaptação aos dados analisados.

A sua característica fundamental é que os primeiros componentes principais da decomposição do sinal em estudo contêm praticamente toda a variância do sinal desejado, sendo os últimos componentes basicamente associados à variância de ruído. Portanto, é possível eliminar esse ruído pela reconstrução do sinal sem os componentes finais.

Como pôde ser visto nos sinais reconstruídos apresentados no trabalho, conclui-se que o método realmente é eficiente para a remoção do ruído 
causado pela baixa amplitude do sinal eletroquímico, não ocasionando adversidades aparentes aos dados de interesse para análise de resultados.

\section{Agradecimentos}

Gostaríamos de agradecer à CAPES e ao CNPq pelo apoio fomentando a pesquisa na universidade $\mathrm{e}$ fornecendo bolsas de mestrado e pós-doutorado, sendo essenciais para o bom desenvolvimento deste trabalho.

\section{Referências}

ALLEN, M. R.; SMITH, L. A. Monte Carlo SSA: detecting irregular oscillations in the Presence of Coloured Noise. Journal of Climate, Boston, n.9, p.3373-3404, 1996.

BENTO, F. R.; MASCARO, L. H. Analysis of the initial stages of electrocrystallization of $\mathrm{Fe}, \mathrm{Co}$ and $\mathrm{Fe}$-Co alloys in chloride solutions. Journal of the Brazilian Chemical Society, São Paulo, v.13, n.4, p.502-509, 2002.

BOCKRIS, J. O. M.; RAZUMNEY, G. A. Fundamental aspects of electrocrystallization. New York: Plenum Press, 1967.

BROOMHEAD, D. S.; KING, G. P. Extracting qualitative dynamics from experimental data. Physica D, Nonlinear Phenomena, Amsterdam, v.20, n.2-3, p.217-236, 1986.

CERQUEIRA, E. O.; POPPI, R. J.; KUBOTA, L. R.; MELLO, C. Utilização de filtro de transformada de fourier para a minimização de ruídos em sinais analíticos. Química Nova, São Paulo, v.23, n.5, p.690-698, 2000.

CHOW, C. W. K.; DAVETY, D. E.; MULCAHY, D. E.; YEOW, T. C. W. Signal enhancement of potentiometric stripping analysis using digital signal processing. Analytica Chimica Acta, Amsterdam, n.307, p.15-26, 1995.

GALVÃO, R. K. H.; ARAÚJO, M. C. U. de; SALDANHA, T. C. B.; VISANI, V.; PIMENTEL, M. F.

Estudo comparativo sobre filtragem de sinais instrumentais usando transformadas de Fourier e Wavelet. Química Nova, São Paulo, v.24, n.6, p.874-884, 2001.

GHIL, M.; ALLEN, M. R.; DETTINGER, M. D.; IDE, K.; KONDRASHOV, D.; MANN, M. E.; ROBERTSON, A. W.; SAUNDERS, A.; TIAN, Y.; VARADI, F.; YIOU, P. Advanced spectral methods for climatic time series. Reviews of Geophysics, Washington, v.40, n.1, p.1.1-1.41, 2002.
GHIL, M.; KEPPENNE, C. L. Adaptive Filtering and Prediction of the Southern Oscillation Index. Journal of Geophysical Research, Washington, v.97, n.d18, p.2044920454, 1992.

OPPENHEIM, A. V.; SCHAFER, R. W.; BUCK, J. R. Discrete-Time Signal Processing. 2. ed. New Jersey: Prentice Hall Signal Processing Series, 1999.

PLETCHER, D. Microelectrodes: theory and applications. Netherlands: Academic Publishers, 1991.

ROBINSON, R. S.; MCCREERY, R. L. Absorption Spectroelectrochemistry with Microelectrodes. Analytical Chemistry, Washington, v.53, n.7, p. 997-1001, 1981.

SCHARIFKER, B.; HILLS, G. Electrochemical Kinetics At Microscopically Small Electrodes . Journal of Electroanalytical Chemistry, Lausanne, v.130, n.1/3, p.8197, 1981.

SILVA, S.M.; ALVES, C.R.; MACHADO, S.A.S; MAZO, L.H.; AVACA, L.A. Electrochemical Determination of Nitrites in Natural Waters with Ultramicroelectrodes. In: MEETING OF INTERNATIONAL SOCIETY OF ELECTROCHEMISTRY, 45 ${ }^{\text {rd }}$, 1994, Porto. Abstracts.... Porto: ISE, 1994. pOVI2.

SOUTHAMPTON ELECTROCHEMISTRY GROUP. Instrumental methods in electrochemistry. England: Ellis Horwood Limited, 1995. p.283-316.

TAVARES, M. C. MACHADO, S.A.S.; MAZO, L.H.; AVACA, L.A.. Hydrogen evolution reaction on $\mathrm{Pt}$ ultramicroelectrodes. In: MEETING OF INTERNATIONAL SOCIETY OF ELECTROCHEMISTRY, 43rd, Cordoba, 1992. Abstracts ... Cordoba: ISE, 1992. p.523.

VAUTARD, R.; YIOU, P.; GHIL, M. Singular-spectrum analysis: a toolkit for short, noisy chaotic signals. Physica D, Nonlinear Phenomena, Amsterdam, v.58, n.1-4, p.95126, 1992.

WIGHTMAN, R. M.; EWING, A. G.; DAYTON, M. A. Pulse Voltammetry with Microvoltammetric Electrodes. Analytical Chemistry, Washiongton, v.53, p.1842-1847, 1981.

WIPF, D. O.; WIGHTMAN, R. M. Voltammetry Under Linear-Diffusion Conditions in Resistive Solvents. Analytical Chemistry, Washiongton, v.62, p.98-102, 1990. 
